\title{
Advice Generation using Fuzzy Logic in OMR Pheonix Technique
}

\author{
Astha Gupta \\ Krishna Engineering College Ghaziabad, India
}

\author{
Niraj Singhal \\ Shobhit University Meerut, India
}

\begin{abstract}
Optical Mark Recognition is the technique used to scan a marked paper to detect the presence or absence of the mark in a predetermined position. Problem with the OMR technique lies is that, it cannot process thin papers and low printing precision answer sheets. OMR Pheonix technique overcomes the limitations of OMR process that were presented in our previous paper [8]. It scans the hard copies of papers which are designed and marked in a specific format and saves that scanned copy in JPEG format, then identifies the responses marked from the JPEG image and stores the result in a database. This paper aims to provide the concept of Fuzzy logic based Advice generator which would be used to give advice to the faculty on the basis of feedback (taken using OMR Pheonix technique) provided by the students.
\end{abstract}

\section{Keywords}

Optical Mark Recognition, Fuzzy Logic, Advice Generation.

\section{INTRODUCTION}

OMR technology can be used when a large volume of data is to be collected and processed in a short period of time or data are to be collected from a large number of sources simultaneously such as in community surveys, consumer surveys, test, assessments, evaluation, feedback, lotteries, voting etc. Questionnaires mainly comprise the selection of categories or "tick box" answer to multiple choice questions.

Some limitations of the current OMR technology are that it requires different hardware and a specific type of paper for scanning. Moreover it is not a good solution for small scale companies or organizations and as the reader is quite sensitive the forms should be in perfect condition, if they are crushed or even folded they may be rejected by the Optical Mark Reader.

OMR pheonix is a low cost solution of OMR process. It neither requires a different hardware for scanning nor a specific type of paper. This is an image based technique which can be used in small scale industries, institutes and schools. In institutes and schools feedback of faculty members can be taken using OMR Pheonix. Feedback analysis can be done using fuzzy logic. The concept of Fuzzy Logic was conceived at the beginning of the 70s by Lotfi Zadeh, a professor at the University of California at Berkley, and presented not as a control methodology, but as a way of processing data by allowing partial set membership rather than crisp set membership or non-membership.

Fuzzy Logic is a problem-solving control system methodology that lends itself to implementation in systems ranging from simple, small, embedded micro-controllers to large, networked, multi-channel PC or workstation-based data acquisition and control systems. It can be implemented in hardware, software, or a combination of both. Fuzzy Logic provides a simple way to arrive at a definite conclusion based upon vague, ambiguous, imprecise, noisy, or missing input information. Fuzzy Logic's approach to control problems mimics how a person would make decisions, only much faster.

We use fuzzy logic in feedback analysis. A set of questions about every faculty member is given to each student. Each individual question is answered in one of linguistic variables (good, ok, poor). All questions are grouped under four categories. For each category, rules have been written to obtain the fuzzified outputs. Fuzzification and defuzzification is done according to mamdani rule based model.

\section{RELATED WORK}

Fuzzy Logic was initiated in 1965 [1,2,3] by Lotfi A. Zadeh, professor for computer science at the University of California in Berkeley. Basically, Fuzzy Logic is a multi-valued logic, that allows intermediate values to be defined between conventional evaluations like true/false, yes/no, high/low, etc. Notions like rather tall or very fast can be formulated mathematically and processed by computers, in order to apply a more human-like way of thinking in the programming of computers [4]. Fuzzy systems are an alternative to traditional notions of set membership and logic that has its origins in ancient Greek philosophy. The precision of mathematics owes its success in large part to the efforts of Aristotle and the philosophers who preceded him. In their efforts to devise a concise theory of logic, and later mathematics, the so-called "Laws of Thought" were posited [5]. One of these, the "Law of the Excluded Middle," states that every proposition must either be True or False. Even when Parminedes proposed the first version of this law (around 400 B.C.) there were strong and immediate objections: for example, Heraclitus proposed that things could be simultaneously True and not True. It was Plato who laid the foundation for what would become fuzzy logic, indicating that there was a third region (beyond True and False) where these opposites "tumbled about." Other, more modern philosophers echoed his sentiments, notably Hegel, Marx, and Engels. But it was Lukasiewicz who first proposed a systematic alternative to the bi-valued logic of Aristotle [6]. Even in the present time some Greeks are still outstanding examples for fussiness and fuzziness. Fuzzy Logic has emerged as a profitable tool for the controlling and steering of systems and complex industrial processes, as well as for household and entertainment electronics, as well as for other expert systems and applications.

We can introduce basic operations on fuzzy sets. Similar to the operations on crisp sets we also want to intersect, unify and negate fuzzy sets. L. A. Zadeh [1] suggested the minimum operator for the intersection and the maximum operator for the union of two fuzzy sets. It can be shown that these operators coincide with the crisp unification, and 
intersection if we only consider the membership degrees 0 and 1. For example, if A is a fuzzy interval between 5 and 8 and B be a fuzzy number about 4 as shown in the Figure below

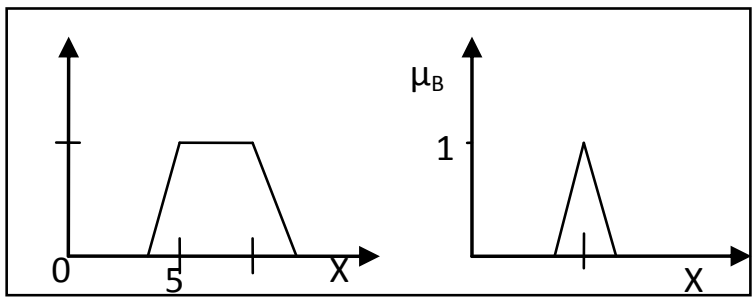

Figure: 1 Example fuzzy sets

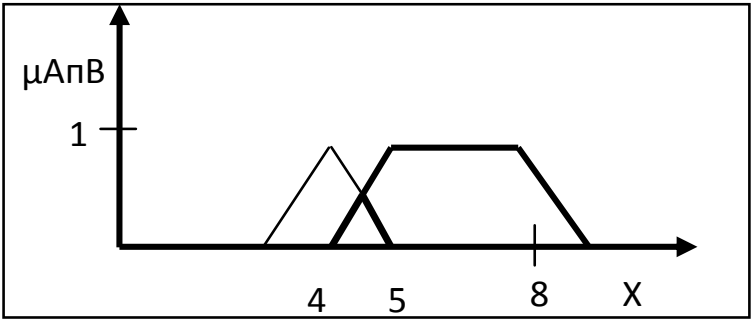

Figure: 2 Fuzzy AND

Fuzzy set between 5 and 8 OR about 4 is shown in the next figure:

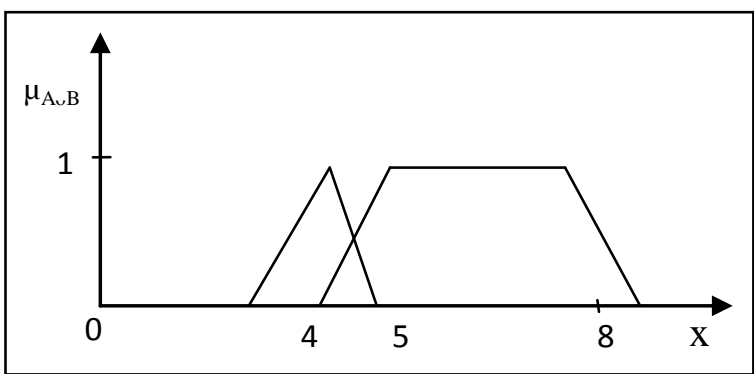

Figure: 3 Fuzzy OR

The NEGATION of the fuzzy set A is shown below:

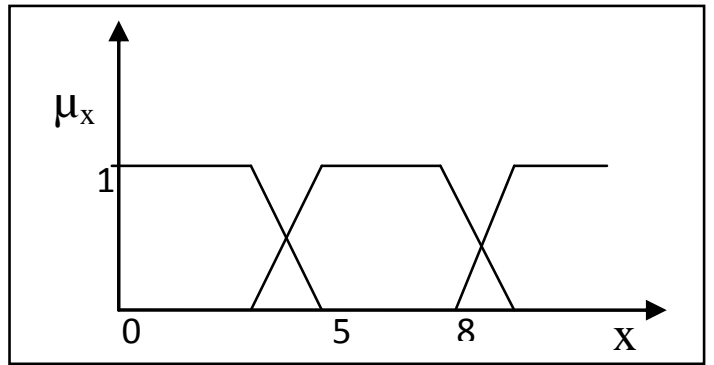

Figure: 4 Fuzzy NEGATIONS

\section{OMR PHEONIX}

Proposed OMR pheonix is a low cost solution of OMR process. It neither requires a different hardware for scanning nor a specific type of paper. This is an image based technique which can be used in small scale industries, institutes and schools.

There are four basic steps to OMR Pheonix:

(i) Design the template. (ii) Image capturing.

(iii) Perform 2D transformation and scaling.

(iv) Find the marks on questionnaire.

\subsection{Template Design}

In this phase, a format of the questionnaire is determined, a template is designed, and whose all coordinates are stored in database. All questionnaire coordinates are matched against this template. A sample of template is shown in figure 5 .

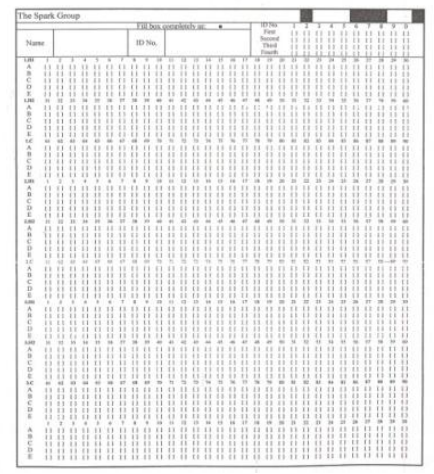

Figure: 5 Layout of questionnaire

The designed template has a grid which helps in checking the alignment and scaling. The coordinates of the top left corner, top right corner, bottom left corner and the bottom right corner of grid are saved. Also the aspect ratios of all the option fields are consistent.

\subsection{Image Capturing}

To capture the image of the questionnaire, flatbed scanners are used. These scanners allow users to work with prints, paper documents, and even three dimensional objects. Normally, the flatbed scanners take 10-20 seconds to scan an $8 \times 10$ inch image. This image is stored in JPEG format

\subsection{Perform Transformation and Scaling}

During the scanning process, if the form is not put properly on the scanner, or if the resolution of the scanner is not set to normal, the scanned image may be tilted or translated with respect to the original template referring figure 6 . This might also happen while photocopying the form (to get multiple copies of blank form). Such issues need to be resolved.

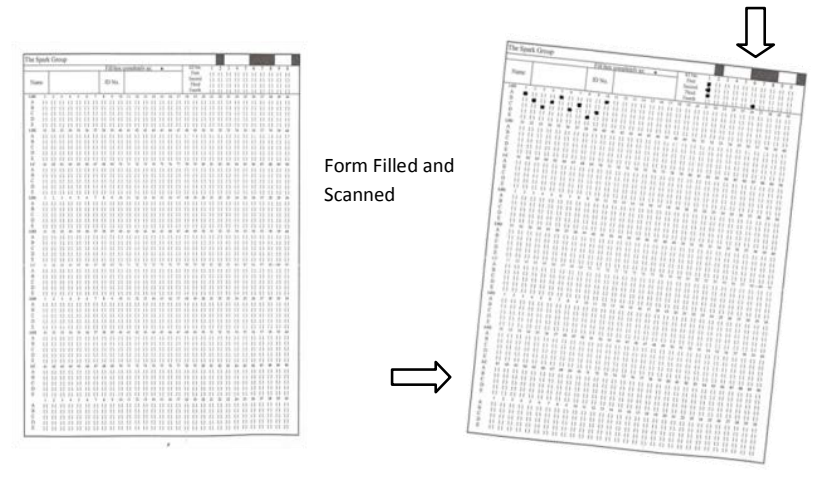

Figure: 6 Scaling and Rotation Issues 


\subsection{Finding Marks}

After the coordinates of the template and questionnaire are matched, marks recognition becomes an easy task. To check whether the mark is filled or not, simple inbuilt functions are used. More than $70 \%$ random pixels of questionnaire are checked. If $50 \%$ or more pixel coordinates are black then the mark is assumed to be "filled". commercial scanners can be used for scanning purpose. Efficiency is comparable to old commercial OMR.

\section{FEEDBACK ANALYSIS}

The OMR Pheonix technique is used in taking feedbacks from students. Analysing the feedback using fuzzy logic a concept of Advice generator is given which would be used to give advice to the faculty on the basis of feedback provided by the students. Two steps should be followed for generating advice:
(i) Rule Formulation
(ii) Advice Generation

\subsection{Rule Formulation}

All the questions are grouped under four different categories, namely Course Organization, Overall Examination Management, Presentation of Course and Interaction with students. For each category, rules have been written to obtain the fuzzified outputs, inputs being the membership classes which are concluded from the weighted scores of individual questions. Apart from the given categories, some extra rules have been formulated based upon the weighted mean to generate a comprehensive advice. Rules for each category are given as under:

\section{Category: Course Organization}

Questions under this category are:

Ques1: Objectives and Plan of the course was specified: Ques2: Coverage and depth of the course plan was: Ques3: The Topic provided new knowledge:

Table: 1 Rules for category-Course Organization

\begin{tabular}{|l|l|l|l|}
\hline $\begin{array}{l}\text { Class } \\
(\text { Ques1 })\end{array}$ & $\begin{array}{l}\text { Class } \\
(\text { Ques2 })\end{array}$ & $\begin{array}{l}\text { Class } \\
(\text { Ques3) }\end{array}$ & $\begin{array}{l}\text { Class } \\
\text { (Output })\end{array}$ \\
\hline Good & Good & Good & Good \\
\hline Good & OK & Good & Good \\
\hline Good & OK & OK & Good \\
\hline Good & Good & OK & Good \\
\hline OK & Good & Good & Good \\
\hline Good & Good & Poor & Good \\
\hline OK & Good & Poor & Good \\
\hline OK & OK & Good & Good \\
\hline OK & OK & OK & OK \\
\hline OK & Poor & OK & OK \\
\hline OK & Good & OK & OK \\
\hline OK & OK & Poor & OK \\
\hline Good & Poor & OK & OK \\
\hline Good & OK & Poor & OK \\
\hline Good & Poor & Good & OK \\
\hline OK & Poor & Good & OK \\
\hline Poor & Good & Good & OK \\
\hline Poor & Good & OK & OK \\
\hline
\end{tabular}

\begin{tabular}{|l|l|l|l|}
\hline Poor & OK & Good & OK \\
\hline Poor & OK & OK & OK \\
\hline OK & Poor & Poor & Poor \\
\hline Poor & Poor & Poor & Poor \\
\hline Poor & OK & Poor & Poor \\
\hline Poor & Poor & OK & Poor \\
\hline Poor & Good & Poor & Poor \\
\hline Poor & Poor & Good & Poor \\
\hline Poor & OK & OK & Poor \\
\hline
\end{tabular}

Category: Overall Examination Management

Questions under this category are:

Ques1: Examination were of appropriate level/length: Ques2: Answer scripts were properly checked and reviewed: Ques3: The grading was fair and transparent:

Table: 2 Rules for category-Overall Examination Management

\begin{tabular}{|c|c|c|c|}
\hline $\begin{array}{c}\text { Class } \\
\text { (Ques1 })\end{array}$ & $\begin{array}{c}\text { Class } \\
\text { (Ques2) }\end{array}$ & $\begin{array}{c}\text { Class } \\
(\text { Ques3) }\end{array}$ & $\begin{array}{c}\text { Class } \\
\text { (Output) }\end{array}$ \\
\hline Good & Good & Good & Good \\
\hline Good & Good & OK & Good \\
\hline Good & OK & Good & Good \\
\hline Good & Poor & Good & Good \\
\hline OK & OK & Good & Good \\
\hline OK & Good & Good & Good \\
\hline OK & Poor & Good & Good \\
\hline Good & Good & Poor & OK \\
\hline Good & OK & OK & OK \\
\hline Good & Poor & OK & OK \\
\hline OK & OK & OK & OK \\
\hline OK & OK & Poor & OK \\
\hline OK & Good & OK & OK \\
\hline OK & Poor & OK & OK \\
\hline Poor & Good & Good & OK \\
\hline Poor & OK & Good & OK \\
\hline Poor & OK & OK & OK \\
\hline Good & Poor & Poor & Poor \\
\hline Good & OK & Poor & Poor \\
\hline OK & Good & Poor & Poor \\
\hline OK & Poor & Poor & Poor \\
\hline Poor & Poor & Poor & Poor \\
\hline Poor & Poor & Good & Poor \\
\hline Poor & Poor & OK & Poor \\
\hline Poor & Good & Poor & Poor \\
\hline Poor & Good & OK & Poor \\
\hline OK & Poor & OK & Poor \\
\hline
\end{tabular}

Category: Presentation of course

Questions under this category are:

Ques1: In terms of organization, clarity and presentation of fundamental concepts, the lectures were:

Ques2: Instructor's oral presentation in terms of audibility and articulation was:

Ques3: Instructor's blackboard presentation in terms of organization and legitibility was: 
Table: 3 Rules for category-Presentation of course

\begin{tabular}{|c|c|c|c|}
\hline $\begin{array}{c}\text { Class } \\
\text { Ques1) }\end{array}$ & $\begin{array}{c}\text { Class } \\
\text { (Ques2) }\end{array}$ & $\begin{array}{c}\text { Class } \\
\text { (Ques3) }\end{array}$ & Class (Output) \\
\hline Good & Good & Good & Good \\
\hline Good & Good & OK & Good \\
\hline Good & Good & Poor & Good \\
\hline Good & OK & Good & Good \\
\hline Good & OK & OK & OK \\
\hline Good & OK & Poor & OK \\
\hline Good & Poor & Good & OK \\
\hline Good & Poor & OK & OK \\
\hline Good & Poor & Poor & Poor \\
\hline OK & Good & Good & OK \\
\hline OK & Good & OK & OK \\
\hline OK & Good & Poor & OK \\
\hline OK & OK & Good & Poor \\
\hline OK & OK & OK & OK \\
\hline OK & OK & Poor & OK \\
\hline OK & Poor & Good & Good \\
\hline OK & Poor & OK & Good \\
\hline OK & Poor & Poor & Good \\
\hline Poor & Good & Good & Poor \\
\hline Poor & Good & OK & Poor \\
\hline Poor & Good & Poor & Poor \\
\hline Poor & OK & Good & OK \\
\hline Poor & OK & OK & OK \\
\hline Poor & OK & Poor & Poor \\
\hline Poor & Poor & Good & OK \\
\hline Poor & Poor & OK & Poor \\
\hline Poor & Poor & Poor & OK \\
\hline & & & \\
\hline
\end{tabular}

Category: Interaction with students

Questions under this category are:

Ques1: Encouragement given by the instructor to think and reason, logically and objectively was:

Ques2: Instructor's response to the questions asked in the class was:

Table: 4 Rules for category-Interaction with students

\begin{tabular}{|c|c|c|}
\hline $\begin{array}{c}\text { Class } \\
\text { (Ques1) }\end{array}$ & $\begin{array}{c}\text { Class } \\
\text { (Ques2) }\end{array}$ & $\begin{array}{c}\text { Class } \\
\text { (Output) }\end{array}$ \\
\hline Good & Good & Good \\
\hline Good & Poor & OK \\
\hline Good & OK & Good \\
\hline OK & Good & Good \\
\hline OK & Poor & Poor \\
\hline OK & OK & OK \\
\hline Poor & Good & OK \\
\hline Poor & Poor & OK \\
\hline Poor & OK & Poor \\
\hline
\end{tabular}

\subsection{Advice Generation}

After the result gets stored in the database, it is retrieved to generate advice. To do so, following steps are followed:

1. Weighted mean of the responses for the individual questions is calculated.
2. For each question the weighted mean is used to obtain the membership of the three parameters (good, ok, poor) i.e. the inputs are fuzzified using Mamdani model [9].

3. Similar questions are grouped together and their combined membership determines the rules which will be fired and their firing strength to generate the fuzzified output.

4. The output variables must have a single membership. Finally defuzzification is done using the weighted means method.

\section{DISSCUSSION}

Generally in institutes and colleges feedbacks are taken on the printed paper manually and feedback analysis is also done manually. There was no technique available for taking feedback and generate advice on basis of it. But now Feedbacks of faculty members can be taken by the OMRPheonix technique and analysis can be done through the concept of fuzzy logic based advice generation technique.

\section{CONCLUSION \& FUTURE SCOPE}

The application developed will automate the analysis of feedback forms in the college and institutes. The whole work consists of three modules. The first one implements an efficient, accurate and robust software based solution to OMR technology. The input form to be used is to be printed on an A4 sheet just once and then can be photocopied to get multiple copies which make it even more cost effective. There is no need of an OMR scanner, a normal scanner is used to scan the filled forms. The scanned copies are then used as input to the software. The second module uses the responses obtained for the graphical analysis of the result. Finally, fuzzy logic has been used to generate advice based upon the result.

The use of a predefined template limits the applications of the software. The future work may include a functionality to generate the template on the fly. Also, the application cannot process all the forms (having different course number and faculty) simultaneously. This limitation can be done away with making use of OCR technology to read the faculty_id and course_id from the form itself. Also barcode technology can be implemented to verify the authenticity of the form. The advice generation part can be made more exhaustive by including features such as comparison of performances of a teacher year wise or subject wise.

process all the forms simultaneously. This limitation can be done away with making use of OCR technology to read the student id from the form itself. Also barcode technology can be implemented to verify the authenticity of the form.

\section{REFERENCES}

[1] L.A. Zadeh," Fuzzy Sets, Information and Control”, 1965.

[2] L.A. Zadeh, "Outline of a New Approach to the Analysis of Complex Systems and Decision Processes", 1973.

[3] L.A. Zadeh, "Fuzzy algorithms", Info. \& Ctl., Vol. 12, pp. 94-102, 1968. 
[4] L.A. Zadeh,"Making computers think like people", IEEE. Spectrum, pp. 26-32, 1984.

[5] S. Korner, "Laws of thought", Encyclopedia of Philosophy, Vol. 4, MacMillan, NY, pp. 414$417,1967$.

[6] C. Lejewski, "Jan Lukasiewicz", Encyclopedia of Philosophy, Vol. 5, MacMillan, NY, pp.104-107, 1967.
[7] A. Reigber, "My life with Kostas", unpublished report, Neverending Story Press, 1999.

[8] Astha Gupta, Niraj Singhal, "Developing an image based low cost solution to the OMR process: OMR Pheonix", communicated to International journal of Computer Application, July 2012.

[9] J.yen and R.Langari, "fuzzy logic", prentice hall, 1999.) 